\title{
Research Article Effects of Empty Sites on Cooperation in the Prisoner's Dilemma Game Based on Social Diversity
}

\author{
Wang Liming ${ }^{1}$ and Feng $\mathrm{Wu}^{2}$ \\ ${ }^{1}$ Department of Physics, Langfang Teachers College, Langfang 065000, China \\ ${ }^{2}$ Department of Physics, Tianjin University of Technology, Tianjin 300191, China \\ Correspondence should be addressed to Wang Liming; wlm_shooker@163.com
}

Received 19 July 2013; Revised 30 November 2013; Accepted 3 December 2013; Published 14 January 2014

Academic Editor: Gualberto Solís-Perales

Copyright (c) $2014 \mathrm{~W}$. Liming and F. Wu. This is an open access article distributed under the Creative Commons Attribution License, which permits unrestricted use, distribution, and reproduction in any medium, provided the original work is properly cited.

\begin{abstract}
We study the effects of empty sites in the prisoner's dilemma game based on social diversity by introducing some empty sites into a square lattice. The results reveal that the empty sites dramatically enhance the cooperation level for a wide range of temptation to defection values if two types of players coexist. By calculating the chances of different type-combinations of the players located on the square lattice, we find that there is an intermediate region where five social ranks are induced to satisfy the certain rank distributions and the cooperation level is significantly enhanced. Moreover, simulation results also show that the moderate gaps among the social ranks can favor cooperation for a larger occupation density.
\end{abstract}

\section{Introduction}

Cooperation is fundamental to biological and social systems. Thus, it is a crucial issue to find and understand what kinds of factors facilitate cooperation. Over the past decades, various versions of evolutionary games have been studied extensively to explore the possibilities for enhancing the cooperative behavior among selfish individuals. Consequently, different mechanisms, for example, kin selection [1], direct [2] and indirect [3] reciprocity, spatial reciprocity [4], voluntary participation [5], and chaotic variations to the payoffs [6], are found to urge the emergence of cooperative behavior in biological systems as well as within human societies [7]. Among all the above mechanisms, the spatial reciprocity has not become an active and important topic until Nowak and May [8] introduced their seminal theoretical mechanism about game and spatial chaos. In recent years, topological inhomogeneities have been introduced to promote the level of cooperation [9-13]. Some investigations suggested that complex networks were beneficial for the evolution of cooperation if its connectivity structure was similar to that of social networks [14-18]. Besides the topological inhomogeneities, inhomogeneities of individual personality have also been introduced $[19,20]$, because of the fact that inhomogeneities of individual personality are the common features of society. Several authors have reported that some distinguished players have the stronger capacities to spread their own strategies, resulting in the thriving of cooperation when the system consists of two types of players with asymmetric teaching and learning activities [21-27]. For example, Droz et al. [21] suggested that the cooperation could be greatly enhanced if there was a relevant difference of the strategy transfer capability between the influential players and their followers. Szolnoki and Szabó [22] found that cooperation could be supported for those models where a portion of players had stronger activities in spreading their own strategies than their neighbors. Li et al. [23] modified the classical spatial game [8] by dividing the population into different types and found that cooperation was significantly promoted for a wide range of the temptation to defection parameters. The study of disordered environments or diluted lattices is another important method in spatial games. Apart from the previous investigation presented by Vainstein and Arenzon [28], there are many reports about the effects of population density on cooperation in recent years [29-32]. For example, Wang et al. $[29,30]$ found that the percolation threshold of an interaction graph constituted the optimal population density for the evolution of cooperation. Mitteldorf and Wilson [31] found 
that patches of cooperators were more favorable at the high density than patches of defectors if the population density was permitted to fluctuate. Nowak et al. [32] investigated interaction between the individuals inside a region of radius $r$ on a diluted square lattice and found that the polymorphism of cooperators and defectors was persistent if $r$ was not too large.

Despite that several authors [33-35] have reviewed the progress of spatial evolutionary games recently, few reports are related to the elucidation of the effect of population density on cooperation in inhomogeneous society. There are still scarce data about the combined effects of both inhomogeneities and population density on cooperation and further efforts are eagerly required. In order to explore how cooperation is influenced by population density in inhomogeneous society, we study the evolution of cooperation in the prisoner's dilemma game on a square lattice with the periodic boundary conditions. In this model, the empty sites, A players, and B players are randomly located on the sites of the square lattice with different concentrations, respectively.

In this paper, we preliminarily focus on spatial game. Further, we only consider how the population density and the social diversity affect the cooperation between individuals. The paper is organized as follows. At first, the details of the model are presented and the parameters of the model are defined in Section 2. Subsequently, the main simulation results and discussions will be presented in Section 3. Finally, the conclusion is summarized in the last section.

\section{Model}

We consider an evolutionary prisoner's dilemma game on a square lattice with the periodic boundary conditions, where two types of players (A and B) are located on the sites of a square lattice with the different concentrations, respectively. Different from the original version of the prisoner's dilemma based on social diversity [23], we assume that some sites are empty. To describe the occupation of the site $i(1<i<N)$, we take $m_{i}$ to be either 1 , if the site $i$ is occupied, or 0 , otherwise. We define the density of players $\rho=\left(n_{c}+n_{d}\right) / N$, where $n_{c}$ is the number of cooperators and $n_{d}$ is the number of defectors. Initially, the empty sites and two types of players (A and $\mathrm{B}$ ) are distributed randomly and a player is designated as a cooperator or defector with the equal percentage. We define the density of A players, $\mu=n_{A} / N \rho$, where $n_{A}$ is the number of A players. In this model, we only consider the simplest case of pure strategies, cooperation (C) and defection (D), represented by the variable $s_{i}=1$ and 0 , respectively. The neighborhood $V_{i}$ is restricted to the nearest neighbors of site $i$ and self-interactions are excluded.

Each Monte Carlo (MC) simulation procedure has two elementary steps. Firstly, all individuals play the game against all their four nearest neighbors (if present) and collect the payoffs from the combats according to the parameterized payoff matrix suggested by Li et al. [23]. That is to say, when two neighboring players play the game, if they are of the same type (namely, both A or both B), the payoff matrix is set to be $T=b>1, R=1$, and $S=P=0$, while if their types are different, the matrix is scaled by a multiplicative factor $\alpha>1$, and then $T=\alpha \cdot b, R=\alpha$ and $S=P=0$. Evidently, this setting of the payoff matrices can be interpreted as that the efficiency actually is equal to the unity for the interactions between two same-type players, while the efficiency is enhanced to be $\alpha$ for two different-type players.

Secondly, after each full cycle of the game, the player $i$ compares its own payoffs with those of a randomly chosen neighbor $j, j \in V_{i}$. If the site $j$ is not empty, the probability with its strategy changing from $s_{i}$ to $s_{j}$ is given by [36]

$$
W\left(s_{i} \longrightarrow s_{j}\right)=\frac{1}{1+\exp \left[\left(P_{i}-P_{j}\right) / K\right]},
$$

where $K=0.1$ characterizes the uncertainty related to the strategy adoption process, and $P_{i}$ and $P_{j}$ are the payoffs of the player $i$ and the player $j$, respectively. In this way, the global density remains constant since no empty sites are ever filled.

In order to characterize the macroscopic behaviors of the system, we introduce a parameter $\rho_{c}(t)$ to represent the fraction of cooperators at a given time as follows:

$$
\rho_{c}(t)=\frac{1}{N} \sum_{i=1}^{N} s_{i}(t) m_{i}(t)
$$

where $N$ is a total number of sites.

Since we are interested in the long time regime, we define a parameter $\rho_{c}$ to represent the average fraction of cooperators as

$$
\rho_{c}=\left\langle\lim _{t \rightarrow \infty} \rho_{c}(t)\right\rangle,
$$

where $\langle\cdot\rangle$ represents averaging many independent initial states. In this paper, a relative cooperator density $\rho_{c} / \rho$ is calculated and used to characterize the macroscopic behaviors of the system. Thus, $\rho_{c} / \rho=0$ means that the population is fully invaded by the defectors, and $\rho_{c} / \rho=1$, by the cooperators. An intermediate case, for example, $0<\rho_{c} / \rho<1$, means that cooperators and defectors coexist.

Before showing the simulation results, we would like to analyze how the parameters $\mu, \rho$, and $\alpha$ affect the type of our model. Note when $\mu=0$ or 1 , only one type of players is left and the model returns to the spatial game on the diluted square lattices [28-32]. The introduction of social diversity ceases to affect and the model also returns to the spatial game on the diluted square lattices [28-32] when $\alpha$ approaches a unity. Moreover, the introduction of the empty sites ceases to affect and the model returns to the original prisoner's dilemma based on social diversity [23] when $\rho$ approaches a unity.

Results of MC simulations displayed below are obtained on a square lattice with the periodic boundary conditions and the size of $N=200 \times 200$, whereas the stationary density of cooperators is calculated by averaging over $1 \times 10^{5} \mathrm{MC}$ steps after the sufficiently long transients. Moreover, the final results are averaged over up to 15 independent runs for each set of parameter values in order to assure an appropriate accuracy. The system is updated synchronously. 


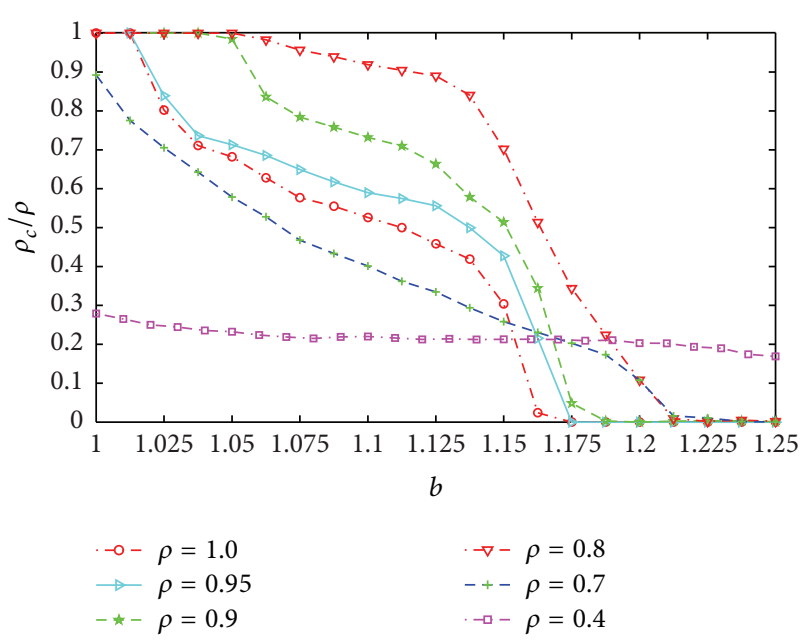

FIGURE 1: Relative density of cooperators $\rho_{c} / \rho$ as a function of temptation to defection $b$ for different values of $\rho$ when $\mu=0.5$ and $\alpha=3$ (see legend). For $\rho=0.9$ or 0.8 , the cooperation level is profoundly enhanced for a wide range of values of $b$. However, the cooperation level is deteriorated for smaller values of $\rho$, for example, $\rho=0.7$. When $\rho=0.4$, relative density of cooperators $\rho_{c} / \rho$ decreases monotonically and slightly with the increase of $b$.

\section{Results and Discussion}

First, we present $\rho_{c} / \rho$ in dependence on $b$ for different values of $\rho$, as shown in Figure 1. For $\rho=1.0$ the model returns to the original prisoner's dilemma based on social diversity [23]. However, in contrast, when some sites on a square lattice are empty, for example, $\rho=0.9$ or 0.8 , the cooperation level is greatly enhanced for a wide range of $b$. It is reasonable for us to infer that there may be the intermediate values of $\rho$ to enhance the cooperation level for a fixed value of $b$.

In order to examine the effects of the parameter $\rho$, we study the relative density of cooperators $\rho_{c} / \rho$ in dependence on $\rho$ for different values of $b$ by setting $\mu=0.5$ and $\alpha=$ 3 , as shown in Figure 2. If the occupation fraction is near 0.05 , almost all players are isolated and do not change their strategies since there is not any combat at all, and the relative density of cooperators is approximately equal to 0.485 . As the density increases, the probability of occurring pairs of occupied sites increases. Irrespective of the value of $b$, the relative density of cooperators $\rho_{c} / \rho$ decreases from around 0.485 , which indicates that some cooperator-defector pairs will become defector-defector pairs. Around $\rho=0.1$, as the number of interacting players increases, the curves for different values of $b$ depart: the higher $b$ is, the better is for defectors. Still further, there are three interesting cases: the first interesting case happens if $b=1.0$, where the relative density of cooperators $\rho_{c} / \rho$ increases monotonically with the increase of $\rho$ until approaching a unity, which indicates the more players there are, the better it is for cooperators. The second interesting case happens if $b$ is in the region $1.05<b \leq 1.175$, for example, $b=1.15$, where the relative density of cooperators increases monotonically with $\rho$ until reaching its maximum value and then sharp decreases. The third interesting case happens if $b$ is in the region $b>1.175$,

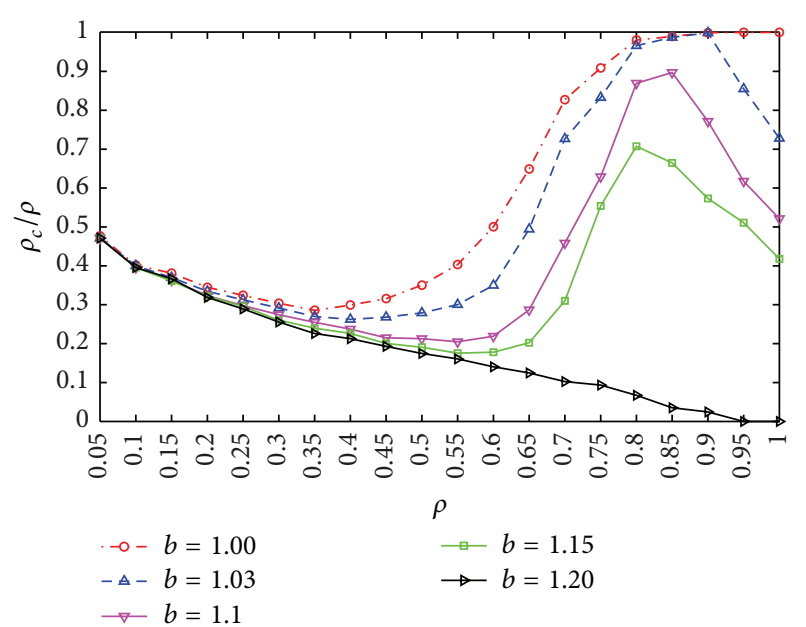

FIGURE 2: Relative density of cooperators $\rho_{c} / \rho$ in dependence on the occupation fraction $\rho$ for different values of $b$ when $\mu=0.5$ and $\alpha=3$ (see legend). Irrespective of the value of $b$, the relative density of cooperators $\rho_{c} / \rho$ decreases from around 0.485 with the increase of $\rho$, and then the curves for different values of $b$ depart around $\rho=0.1$. Still further, there are three interesting cases: the first interesting case occurs if $b=1.0$, where the relative density of cooperators $\rho_{c} / \rho$ increases monotonically with the increase of $\rho$ until approaching a unity; the second interesting case occurs if $b$ is in the region, $1.05<b \leq 1.175$, where the relative density of cooperators increases monotonically with the increase of $\rho$ until reaching its maximum value and then sharp decreases; the third interesting case appears if $b$ is in the region $b>1.175$, for example, $b=1.2$, where the relative density of cooperators decreases monotonically with the increase of $\rho$ until approaching zero.

for example, $b=1.2$, where the behavior is monotonic and the relative density of cooperators decreases monotonically with the increase of $\rho$ until approaching zero. The above results further confirm our previous assertion about some appropriate values for $\rho$ promoting cooperation.

In order to examine the effects of the parameter $\rho$ on the cooperation level, we study the relative density of cooperators $\rho_{c} / \rho$ in dependence on $\rho$ for different values of $\mu$ by setting $\alpha=3.0$ and $b=1.15$, as shown in Figure 3. If the occupation fraction is near 0.06 , almost all players are isolated and do not change their strategies since there is not any combat at all. Irrespective of the values of $\mu$, the relative density of cooperators is approximately equal to 0.47 . As the occupation fraction increases, the probability of occurring pairs of occupied sites increases, and for all the values of $\mu$, the relative fraction of cooperators decreases from around 0.47 , which indicates some cooperator-defector pairs will become defector-defector pairs. Around $\rho=0.1$, as the number of interacting individuals increases, the curves for different values of $\mu$ depart: for $\mu=0$, the model returns to the spatial game on the diluted square lattices [28-32], where the relative density of cooperators decreases monotonically with the increase of $\rho$ until approaching zero. For $\mu \neq 0$, there are two cases: the first case occurs when $\mu$ is equal to 0.1 or 0.2 , where the relative density of cooperators decreases monotonically with the increase of $\rho$ until approaching its minimum value, then increases monotonically with the increase of $\rho$ until 


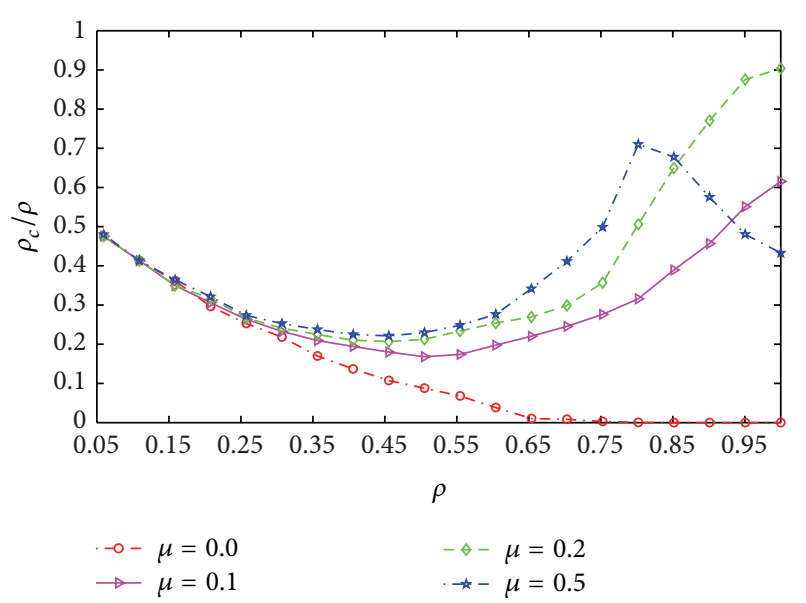

FIGURE 3: Relative density of cooperators $\rho_{c} / \rho$ in dependence on the occupation fraction $\rho$ for the different fractions of A players $\mu$ when $\alpha=3.0$ and $b=1.15$ (see legend). Irrespective of the values of $\mu$, the relative fraction of cooperators decreases from around 0.47 with the increase of $\rho$, and then the curves for different values of $\mu$ depart around $\rho=0.1$. For $\mu=0$, the relative density of cooperators decreases monotonically with the increase of $\rho$ until approaching zero. When $\mu$ is equal to 0.1 or 0.2 , the relative density of cooperators decreases monotonically with the increase of $\rho$ until approaching its minimum value and then increases monotonically with the increase of $\rho$ until approaching its maximum value at $\rho=1.0$. In contrast, when $\mu$ is equal to 0.5 , the relative density of cooperators decreases monotonically with the increase of $\rho$ until approaching its minimum value, then increases monotonically with the increase of $\rho$ until approaching its maximum value at around $\rho=0.8$, and finally sharp decreases with the increase of $\rho$ until approaching its minimum value at $\rho=1.0$.

approaching its maximum value at $\rho=1.0$. The second case happens when $\mu$ is equal to 0.5 , where the relative density of cooperators decreases monotonically with the increase of $\rho$ until approaching its minimum value and then increases monotonically with the increase of $\rho$ until approaching its maximum value around $\rho=0.8$, and finally sharp decreases with the increase of $\rho$ until approaching its minimum value at $\rho=1.0$. The above results indicate that some appropriate values of $\mu$ can promote cooperation.

Besides, for the sake of intuitively understanding the effects of the parameter $\rho$, some typical snapshots of the system at equilibrium are plotted with respect to different values of $\rho$ when $b=1.15, \mu=0.5$, and $\alpha=3.0$, as shown in Figure 4. Of course, Figure 4 further confirms the conclusions drawn from Figure 3.

In order to examine the effects of the parameter $\mu$ on the cooperation level, we study $\rho_{c} / \rho$ independence on $\mu$ for different values of $\rho$ by setting $b=1.15$ and $\alpha=3.0$, as shown in Figure 5. These curves are symmetric around the middle line $\mu=0.5$. For a larger occupation fraction, for example, $\rho=$ 1.0 or 0.9 , the fraction of cooperators increases monotonically with the increase of $\mu$ until reaching its maximum value and then decreases with the increase of $\mu$ until the middle point at $\mu=0.5$. Similar non-monotonic behaviors were also found in the previous two-type-player model [23], where each site on the square lattice was occupied. Moreover, the optimum values that maximize the fraction of cooperators are dependent on the region of $\rho$ and are different. For fixed $b$ and $\alpha$, the curves turn into the unimodal curves from the bimodal curves with the decrease of $\rho$. For example, for $\rho=0.9$, there are two maximum values that approximately occur at $\mu=0.28$ and 0.72 , respectively, but for $\rho=0.8$, there is only one maximum value that approximately occurs at $\mu=0.5$.

In the above discussion, we successively examine the effects of the parameters $b, \rho$, and $\mu$ on the relative density of cooperators, while there is still another parameter $\alpha$ not to be discussed. Thus we now focus on how $\rho_{c} / \rho$ changes with $\alpha$. Results obtained for the different values of $\rho$ by setting $b=$ 1.15 and $\mu=0.5$ are revealed in Figure 6. For comparison, for the extreme case $\rho=1.0$, the results from [23] are also shown in Figure 6. For the lower occupation densities, for example, $\rho=0.4$, all the isolated cooperator clusters are able to survive for all the values of $\alpha$. But interestingly, as soon as $\rho>0.4$, the relative density of cooperators varies nonmonotonically with the increase of $\alpha$, which indicates that moderately enlarging the gaps among the social ranks can facilitate the cooperation, while exceedingly large or small gaps inhibit cooperation. In addition, although cooperation is possible in a wide range of $\alpha$ for $0.6 \leq \rho<1.0$, cooperation performs best when $\rho=0.8$. Thus, the two immediate conclusions are (1) cooperation is possible and enhanced if the empty sites are present and (2) the intermediate values of $\alpha$ enhance cooperation (unless the density $\rho$ is so low that the game between player and its neighbors does not occur), and exceedingly large or small $\alpha$ inhibits cooperation.

In order to investigate the relation between cooperation and social diversity, Perc and Szolnoki [36] introduced the diversity in players' payoffs into the classical spatial prisoner's dilemma game [8] and showed that the diversity could promote cooperation. They also accounted for the observation by the feedback mechanism, in which the players with higher payoffs could dominate their neighbors and eventually form large clusters around them. On the basis of Perc's work, Li et al. [23] divided the people into two types, that is, A and B. They argued that the types of players are actually dummy, and it is the induced social ranks that play an essential role in the facilitation of cooperation. Besides, they also pointed out that the gaps among ranks depend linearly on the factor of efficiency and the definition of social rank makes sense when the factor of efficiency is much larger than 1.

As to the reasons for some appropriate values for $\rho$ promoting cooperation shown in this paper, referring to the previous works by Li et al. [23], we argue that the empty sites not only induce the changes in the diversified social ranks (or the feedback mechanism) but also prohibit defectors to exploit cooperators. To confirm this argument, we should analyze the varied status of the players in our model, where we define the social rank of a player as the number of its differenttype neighbors. When a player plays games with its neighbors, the higher its rank is, the more payoffs obtained in a one-shot game are enhanced by the parameter $\alpha$, and the stronger its ability to collect payoffs. Here, the rank of the player means its ability to collect payoffs. Since the degree of each node on the square lattice is equal to 4 , a player may have $0,1,2$, 


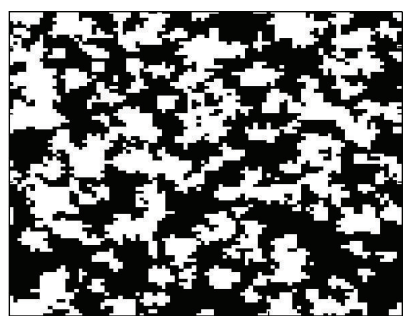

(a)

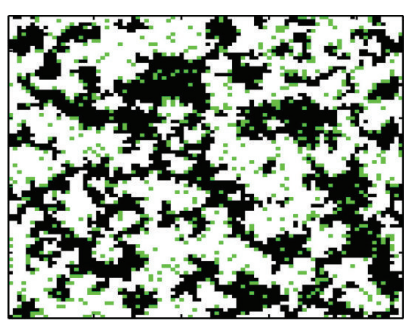

(b)

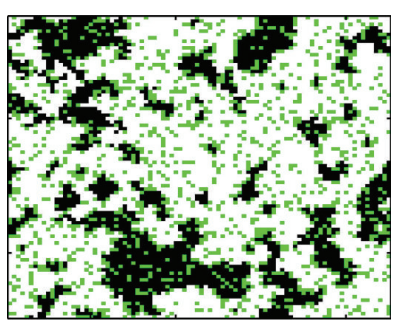

(c)

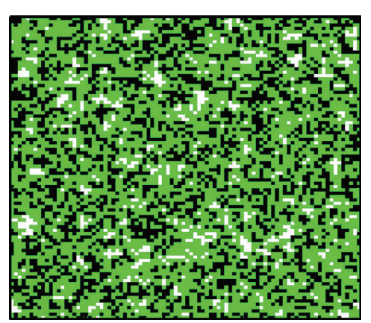

(d)

FIGURE 4: Snapshots of typical distributions of cooperators (white), defectors (black), and empty sites (green) on the square lattice for different values of $\rho$ when $b=1.15, \mu=0.5$, and $\alpha=3.0$ : (a) $\rho=1.0$; (b) $\rho=0.9$; (c) $\rho=0.8$; (d) $\rho=0.4$.

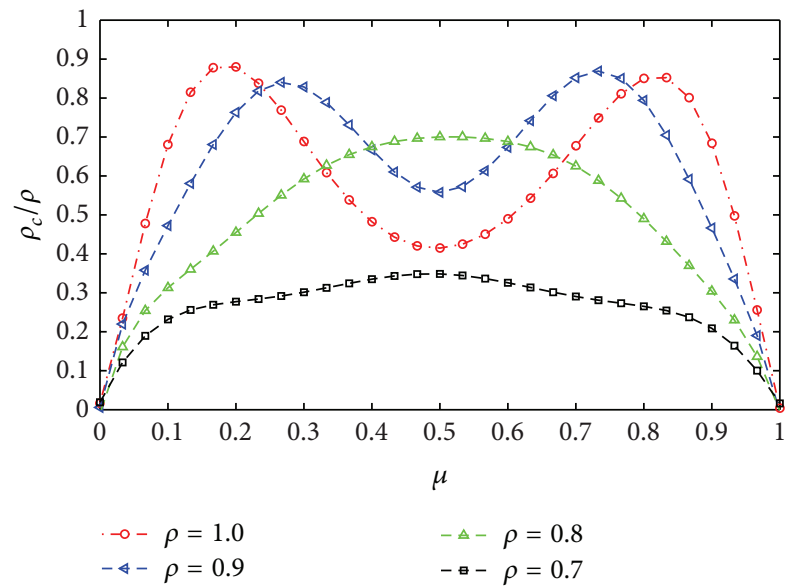

FIGURE 5: Relative density of cooperators $\rho_{c} / \rho$ in dependence on the fraction of A players $\mu$ for different values of $\rho$ when $b=1.15$ and $\alpha=3.0$ (see legend). Depending on the region of $\rho$, the optimum values $\mu$ that maximize the fraction of cooperators are different: for a larger occupation fraction, there are two maximum values. For example, two maximum values approximately occur at $\mu=0.28$ and 0.72 when $\rho=0.9$, respectively. However, for a smaller occupation fraction, for example, $\rho=0.8$ or 0.7 , there is only one maximum value that approximately occurs at $\mu=0.5$.

3, or 4 different-type neighbors obviously, which determines the player's ranks accordingly. Thus, there are five unequal social ranks in our model. When the focal site is not empty, by calculating the chances of different type-combinations of the focal players located on the square lattice, it is easy to give a so-called rank distribution formula for $\mu$ and $\rho$ as follows:

$$
\begin{aligned}
& n_{i}(\mu, \rho)=C_{4}^{i} C_{4-i}^{r}(1-\rho)^{r} \\
& \times\left[(\rho-\mu)^{i} \mu^{5-i-r}+\mu^{i}(\rho-\mu)^{5-i-r}\right],
\end{aligned}
$$

where $i=0,1,2,3,4$, respectively, denote the five ranks in the population, $n_{i}(\mu, \rho)$ denotes the relative fraction of players belonging to rank $i$ for fixed $\mu$ and $\rho$, both $C_{4}^{i}$ and $C_{4-i}^{r}$ are the combination numbers, and $r$ denotes the number of empty sites in a focal player's neighbors. Obviously, (4) has a symmetry about $\mu$ for a fixed $\rho$, because the value of $n_{i}(\mu, \rho)$ should be invariant when we switch each player's type to the other type (meanwhile the value of $\mu$ is changed into $(\rho-\mu)$ ). For the presentation of the direct observation and impression,

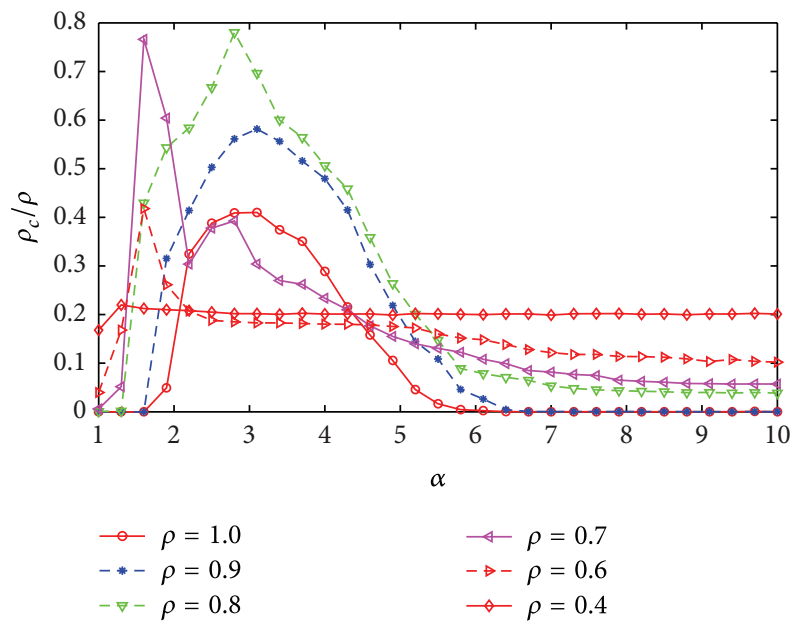

FIGURE 6: Relative density of cooperators $\rho_{c} / \rho$ against $\alpha$ for different values of $\rho$ when $b=1.15$ and $\mu=0.5$ (see legend). For the lower occupation densities, for example, $\rho=0.4$, all isolated cooperator clusters are able to survive for all the values of $\alpha$. But it is worth noting that the relative density of cooperators varies nonmonotonically with the increase of $\alpha$ when $\rho>0.4$, which indicates that moderately enlarging the gaps among the social ranks can facilitate cooperation, while exceedingly large or small gaps inhibit cooperation. Moreover, although cooperation is possible in a wide range of $\alpha$ for $0.6 \leq \rho<1$, cooperation performs best when $\rho=0.8$.

we plot the histograms of the rank distribution for different values of $\rho$ by setting $\mu=0.5$ in Figure 7 .

By virtue of these histograms, one can find that the different rank distributions in Figure 7 actually correspond to the different trends of the curves in Figure 1. When $\rho$ is slightly smaller than a unity, for example, $\rho=0.8$, as shown in Figure 7(b), most players still occupy the three middle ranks, which are similar to those in Figure $7(\mathrm{a})$. Thereby, the feedback mechanism in Figure 7(b) is similar to that in Figure 7(a). Nevertheless, under the rank distribution in Figure 7(b), cooperators not only can utilize the interconnectedness between them to form compact clusters, but also can benefit from the dilution that prohibits defectors to exploit them with the same efficiency as on the square lattice fully occupied by players. Thus, the level of cooperation is substantially enhanced in the region of $1.0125 \leq b \leq$ 1.2125 when $\rho=0.8$, as shown in Figure 1. Especially, when 


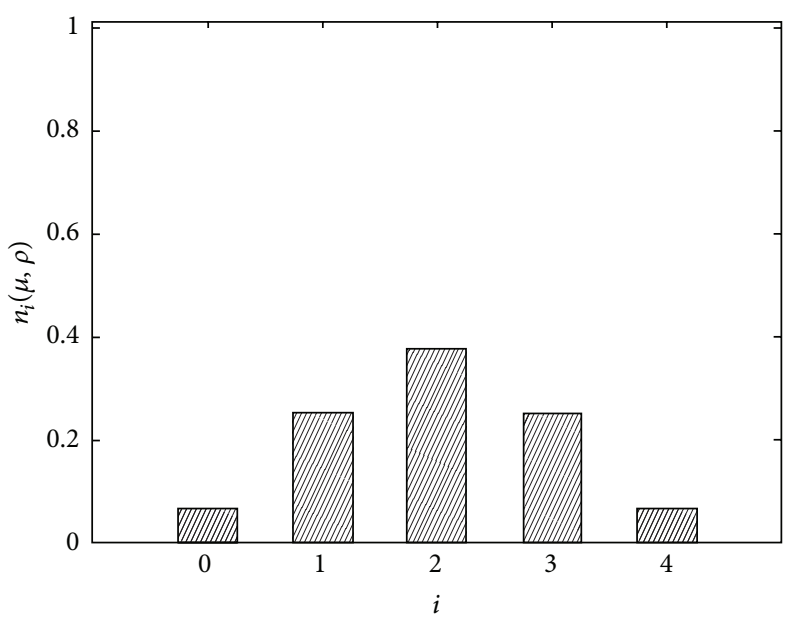

(a)

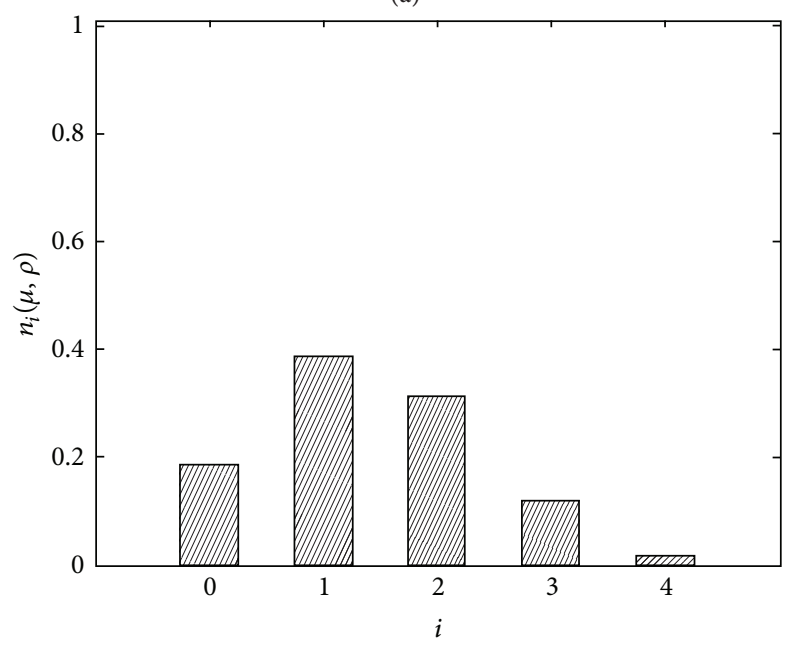

(c)

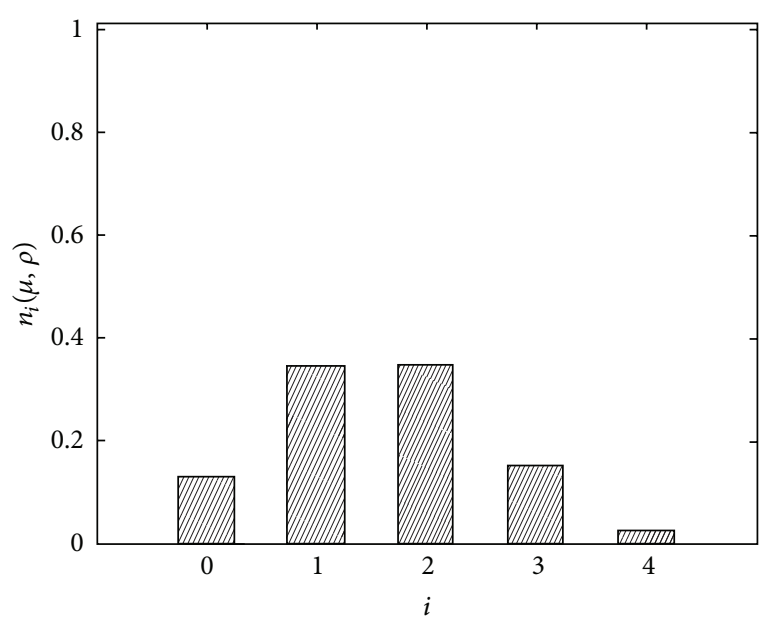

(b)

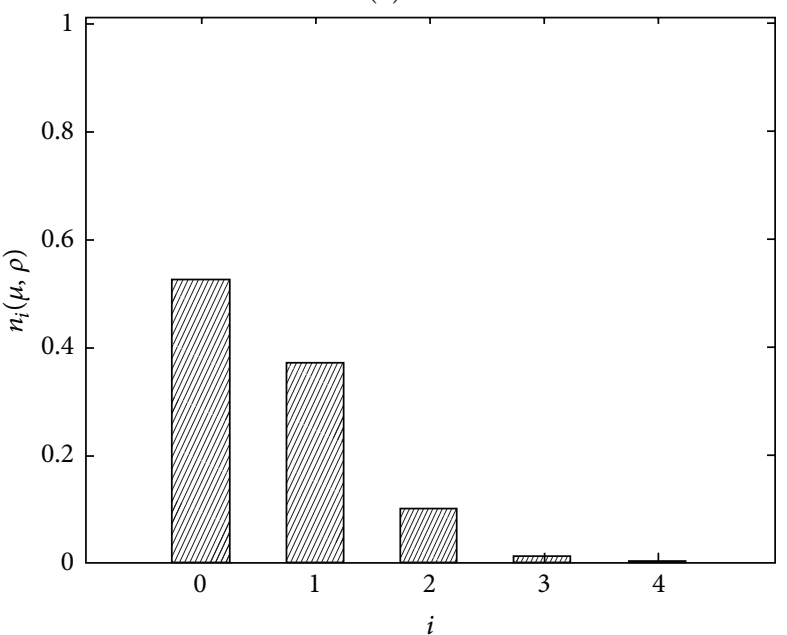

(d)

FIGURE 7: Relative fraction of players belonging to rank $i$ for different values of $\rho$ when $\mu=0.5$ : (a) $\rho=1.0$; (b) $\rho=0.8$; (c) $\rho=0.7$; (d) $\rho=0.4$. For $\rho=0.8$, most players still occupy the three middle ranks, which indicates that the feedback mechanism in (b) is similar to that in (a). Nevertheless, the empty sites prohibit defectors to exploit cooperators, and thus the level of cooperation is substantially enhanced in the region of $1.0125 \leq b \leq 1.2125$ when $\rho=0.8$, as shown in Figure 1. However, for $\rho=0.7$, most players occupy three lower ranks, while only a few players emerge in two higher ranks as shown in (c), which drastically weaken the feedback mechanism. Despite that cooperators may still benefit from the dilution, the level of cooperation remarkably decreases by comparison with that for $\rho=0.8$, as shown in Figure 1 . When $\rho=0.4$, as shown in (d), almost all players occupy three lower ranks and nearly none occupy two higher ranks. This kind of rank distribution results in a further decrease of $\rho_{c} / \rho$, as shown in Figure 1.

$\rho$ further decreases, for example, $\rho=0.7$, as shown in Figure $7(\mathrm{c})$, the rank distribution obviously changes: most players occupy three lower ranks, while only a few players emerge in two higher ranks, which dramatically weakens the feedback mechanism. Despite that cooperators may still benefit from the dilution, the level of cooperation remarkably decreases by comparison with that for $\rho=0.8$, as shown in Figure 1. When $\rho=0.4$, almost all players occupy three lower ranks and nearly none occupy two higher ranks, as shown in Figure 7(d). It is reasonable for us to infer that the feedback mechanism is further weakened due to the decrease of the higher rank players, which results in a further decrease of $\rho_{c} / \rho$, as shown in Figure 1. Moreover, it is found that the smaller $\rho$ is, the more the number of players in lower ranks is, which is consistent with our experience, because a large amount of empty sites reduce the chances of different-type neighbors between the focal player and its neighbors.

Besides, the rank distribution is also affected by $\mu$ for fixed $\rho$, which was investigated in detail [23]. Since the specific rank distribution induced by parameters $\mu$ and $\rho$ has a significant impact on the evolution of the system, in order to understand comprehensively the influence of $\mu$ and $\rho$ on the rank distribution, the surface charts of the rank distribution dependent on $\mu$ and $\rho$ are plotted in Figure 8. Of course, these results further confirm our previous assertion from the histograms.

\section{Conclusions}

In summary, we have extended the model of [23] by introducing some empty sites and endowed this model with more 


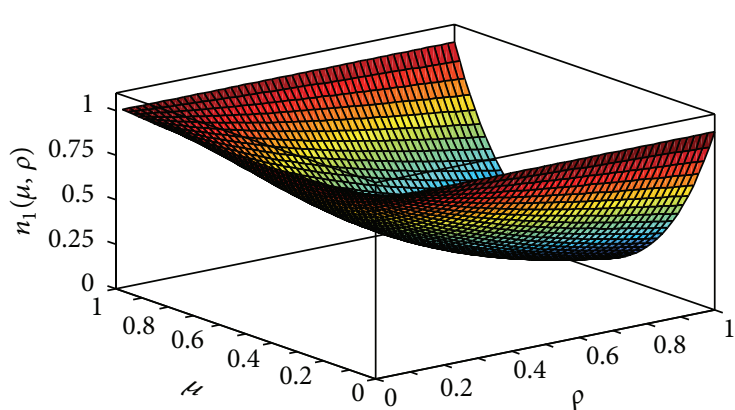

(a)

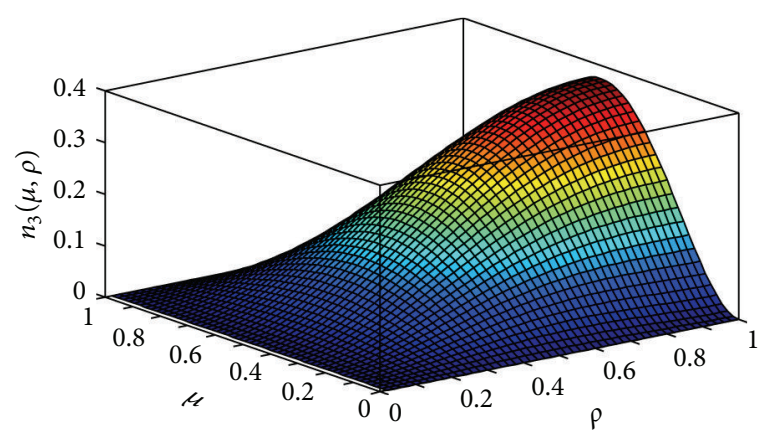

(c)

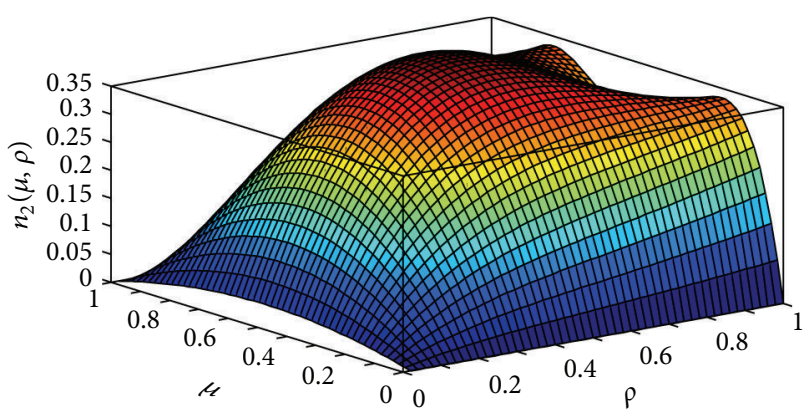

(b)

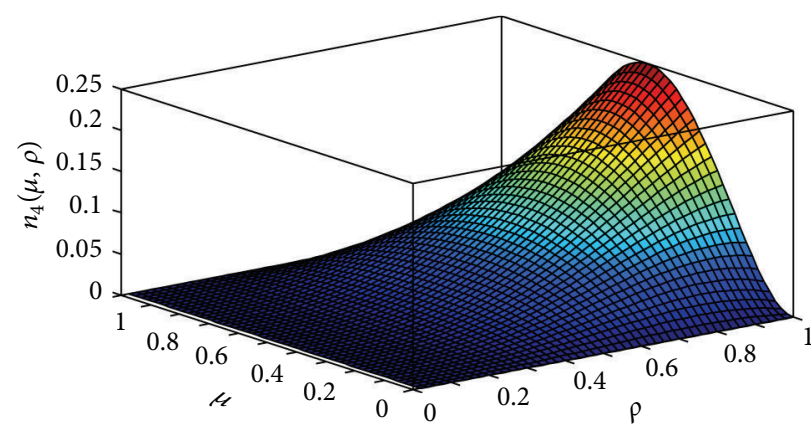

(d)

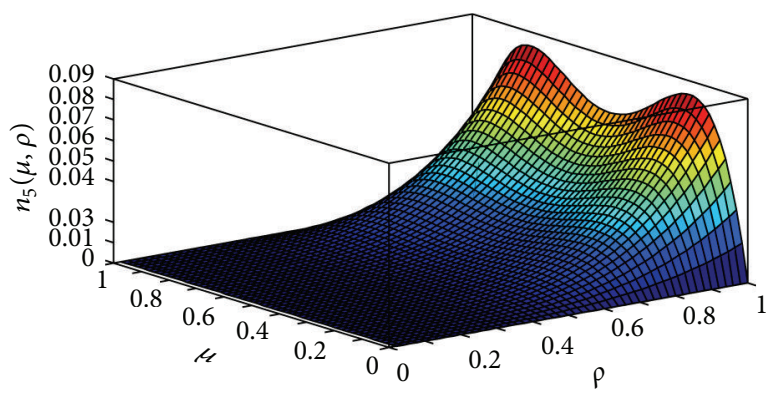

(e)

FIGURE 8: Relative fraction of players belonging to different ranks in dependence on $\mu$ and $\rho$ : (a) $n_{1}(\mu, \rho)$; (b) $n_{2}(\mu, \rho)$; (c) $n_{3}(\mu, \rho)$; (d) $n_{4}(\mu, \rho)$; (e) $n_{5}(\mu, \rho)$. These results confirm that the parameters $\mu$ and $\rho$ have the significant effects on the rank distribution.

realistic meaning. We have investigated the effects of empty sites on cooperation in the prisoner's dilemma game on the square lattice on the base of social diversity, in which five unequal social ranks are induced by both the setup of the two-type players and empty sites. Our results show that the empty sites dramatically enhance the cooperation level for a wide range of $b$ when A players coexist with $B$ players. Moreover, it is found that the maximal cooperation level is obtained when the proportion between the two types of players is quite imbalanced for a larger value of $\rho$, whereas the maximal cooperation level is obtained when the proportion between the two types is quite balanced for a smaller value of $\rho$. These results indicate that the optimal collocation between $\mu$ and $\rho$ substantially promotes the cooperation level. It is also found that the cooperation fraction nonmonotonically relies on the factor $\alpha$ for a larger occupation fraction, which indicates that the moderate gaps among the social ranks can favor cooperation for a larger occupation fraction. Finally, by calculating the chances of different type-combinations of the players located on the square lattice, we provide a rank distribution formula for $\mu$ and $\rho$. Based on the above analysis, we find that there is the intermediate region for $\mu$ and $\rho$ that induces five social ranks satisfying certain rank distributions, which is why the intermediate values for $\mu$ and $\rho$ lead to the highest cooperation level at some fixed values of $b$ and $\alpha$. We hope this work can contribute to understanding the puzzle of the social cooperation. As for the effect of the temporal aspects of the environment on the interactions between individuals, we will discuss it elsewhere.

\section{Conflict of Interests}

The authors declare that there is no conflict of interests regarding the publication of this paper. 


\section{Acknowledgments}

This work was partially supported by the Langfang Teachers College Foundation, China (Grant no. LSZY201204), and the National Science Fund for Distinguished Young Scholars of China (Grant no. 11204214).

\section{References}

[1] W. D. Hamilton, "The genetical evolution of social behaviourI," Journal of Theoretical Biology, vol. 7, no. 1, pp. 1-16, 1964.

[2] R. Axelrod and W. D. Hamilton, "The evolution of cooperation," Science, vol. 211, no. 4489, pp. 1390-1396, 1981.

[3] E. Fehr and S. Gächter, "Altruistic punishment in humans," Nature, vol. 415, no. 6868, pp. 137-140, 2002.

[4] C. Hauert, S. De Monte, J. Hofbauer, and K. Sigmund, "Volunteering as Red Queen mechanism for cooperation in public goods games," Science, vol. 296, no. 5570, pp. 1129-1132, 2002.

[5] M. Perc, "Chaos promotes cooperation in the spatial prisoner's dilemma game," Europhysics Letters, vol. 75, no. 6, pp. 841-846, 2006.

[6] M. A. Nowak and R. M. May, "The spatial dilemmas of evolution," International Journal of Bifurcation and Chaos in Applied Sciences and Engineering, vol. 3, no. 1, pp. 35-78, 1993.

[7] M. A. Nowak and K. Sigmund, "Evolutionary dynamics of biological games," Science, vol. 303, no. 5659, pp. 793-799, 2004.

[8] M. A. Nowak and R. M. May, "Evolutionary games and spatial chaos," Nature, vol. 359, no. 6398, pp. 826-829, 1992.

[9] F. C. Santos and J. M. Pacheco, "Scale-free networks provide a unifying framework for the emergence of cooperation," Physical Review Letters, vol. 95, no. 9, Article ID 098104, 2005.

[10] G. Abramson and M. Kuperman, "Social games in a social network," Physical Review E, vol. 63, no. 3, Article ID 030901, 4 pages, 2001.

[11] E. Lleberman, C. Hauert, and M. A. Howak, "Evolutionary dynamics on graphs," Nature, vol. 433, no. 7023, pp. 312-316, 2005.

[12] W.-X. Wang, J. Ren, G. Chen, and B.-H. Wang, "Memory-based snowdrift game on networks," Physical Review E, vol. 74, no. 5, Article ID 056113, 2006.

[13] X. Chen and L. Wang, "Promotion of cooperation induced by appropriate payoff aspirations in a small-world networked game," Physical Review E, vol. 77, no. 1, Article ID 017103, 2008.

[14] J. Ren, W.-X. Wang, and F. Qi, "Randomness enhances cooperation: a resonance-type phenomenon in evolutionary games," Physical Review E, vol. 75, no. 4, Article ID 045101, 4 pages, 2007.

[15] Z. Rong, X. Li, and X. Wang, "Roles of mixing patterns in cooperation on a scale-free networked game," Physical Review E, vol. 76, no. 2, Article ID 027101, 2007.

[16] M. Tomassini, E. Pestelacci, and L. Luthi, "Social dilemmas and cooperation in complex networks," International Journal of Modern Physics C, vol. 18, no. 7, pp. 1173-1185, 2007.

[17] M. N. Kuperman and S. Risau-Gusman, "The effect of the topology on the spatial ultimatum game," European Physical Journal B, vol. 62, no. 2, pp. 233-238, 2008.

[18] J. Poncela, J. Gómez-Gardeñes, L. M. Floría, and Y. Moreno, "Robustness of cooperation in the evolutionary prisoner's dilemma on complex networks," New Journal of Physics, vol. 9, article 184, 2007.
[19] H. X. Yang, W. X. Wang, Z. X. Wu, Y. C. Lai, and B. H. Wang, "Diversity-optimized cooperation on complex networks," Physical Review E, vol. 79, no. 5, Article ID 056107, 7 pages, 2009.

[20] Y.-Z. Chen, Z.-G. Huang, S.-J. Wang, Y. Zhang, and Y.-H. Wang, "Diversity of rationality affects the evolution of cooperation," Physical Review E, vol. 79, no. 5, Article ID 055101, 2009.

[21] M. Droz, J. Szwabinski, and G. Szabó, "Motion of influential players can support cooperation in Prisoner's Dilemma," European Physical Journal B, vol. 71, no. 4, pp. 579-585, 2009.

[22] A. Szolnoki and G. Szabó, "Cooperation enhanced by inhomogeneous activity of teaching for evolutionary prisoner's dilemma games," Europhysics Letters, vol. 77, no. 3, Article ID 30004, 5 pages, 2007.

[23] Z.-H. Li, B.-H. W, R. - Liu, and H.-X. Yang, "Evolutionary prisoner's dilemma game based on division of work," Chinese Physics Letters, vol. 26, no. 10, Article ID 108701, 4 pages, 2009.

[24] Z.-X. Wu, X.-J. Xu, Z.-G. Huang, S.-J. Wang, and Y.-H. Wang, "Evolutionary prisoner's dilemma game with dynamic preferential selection," Physical Review E, vol. 74, no. 2, Article ID 021107, 7 pages, 2006.

[25] M. Perc, A. Szolnoki, and G. Szabó, "Restricted connections among distinguished players support cooperation," Physical Review E, vol. 78, no. 6, Article ID 066101, 2008.

[26] C.-L. Tang, W.-X. Wang, X. Wu, and B.-H. Wang, "Effects of average degree on cooperation in networked evolutionary game," European Physical Journal B, vol. 53, no. 3, pp. 411-415, 2006.

[27] G. Szabó and A. Szolnoki, "Cooperation in spatial prisoner's dilemma with two types of players for increasing number of neighbors," Physical Review E, vol. 79, no. 1, Article ID 016106, 2009.

[28] M. H. Vainstein and J. J. Arenzon, "Disordered environments in spatial games," Physical Review E, vol. 64, no. 5, Article ID 051905, 6 pages, 2001.

[29] Z. Wang, A. Szolnoki, and M. Perc, "If players are sparse social dilemmas are too: importance of percolation for evolution of cooperation," Scientific Reports, vol. 2, Article ID 00369, 2012.

[30] Z. Wang, A. Szolnoki, and M. Perc, "Percolation threshold determines the optimal population density for public cooperation," Physical Review E, vol. 85, no. 3, Article ID 037101, 2012.

[31] J. Mitteldorf and D. S. Wilson, "Population viscosity and the evolution of altruism," Journal of Theoretical Biology, vol. 204, no. 4, pp. 481-496, 2000.

[32] M. A. Nowak, S. Bonhoeffer, and R. M. May, "Spatial games and the maintenance of cooperation," Proceedings of the National Academy of Sciences of the United States of America, vol. 91, no. 11, pp. 4877-4881, 1994.

[33] M. Perc, J. G. Gómez-Gardeñes, A. Szolnoki, L. M. Floría, and Y. Moreno, "Evolutionary dynamics of group interactions on structured populations: a review," Journal of the Royal Society Interface, vol. 10, no. 80, Article ID 0907, 17 pages, 2013.

[34] M. Perc and P. Grigolini, "Collective behavior and evolutionary games-an introduction," Chaos, Solitons \& Fractals, vol. 56, pp. $1-5,2013$.

[35] M. Perc and A. Szolnoki, "Coevolutionary games-A mini review," BioSystems, vol. 99, no. 2, pp. 109-125, 2010.

[36] M. Perc and A. Szolnoki, "Social diversity and promotion of cooperation in the spatial prisoner's dilemma game," Physical Review E, vol. 77, no. 1, Article ID 011904, 2008. 


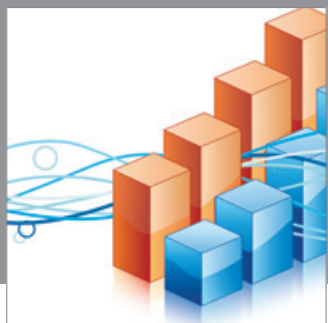

Advances in

Operations Research

mansans

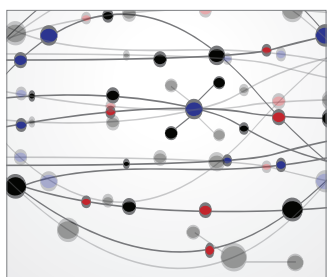

The Scientific World Journal
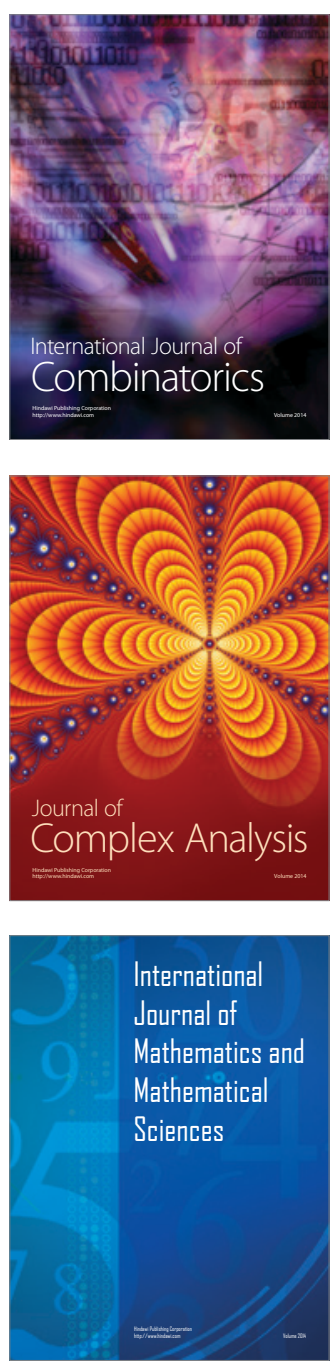
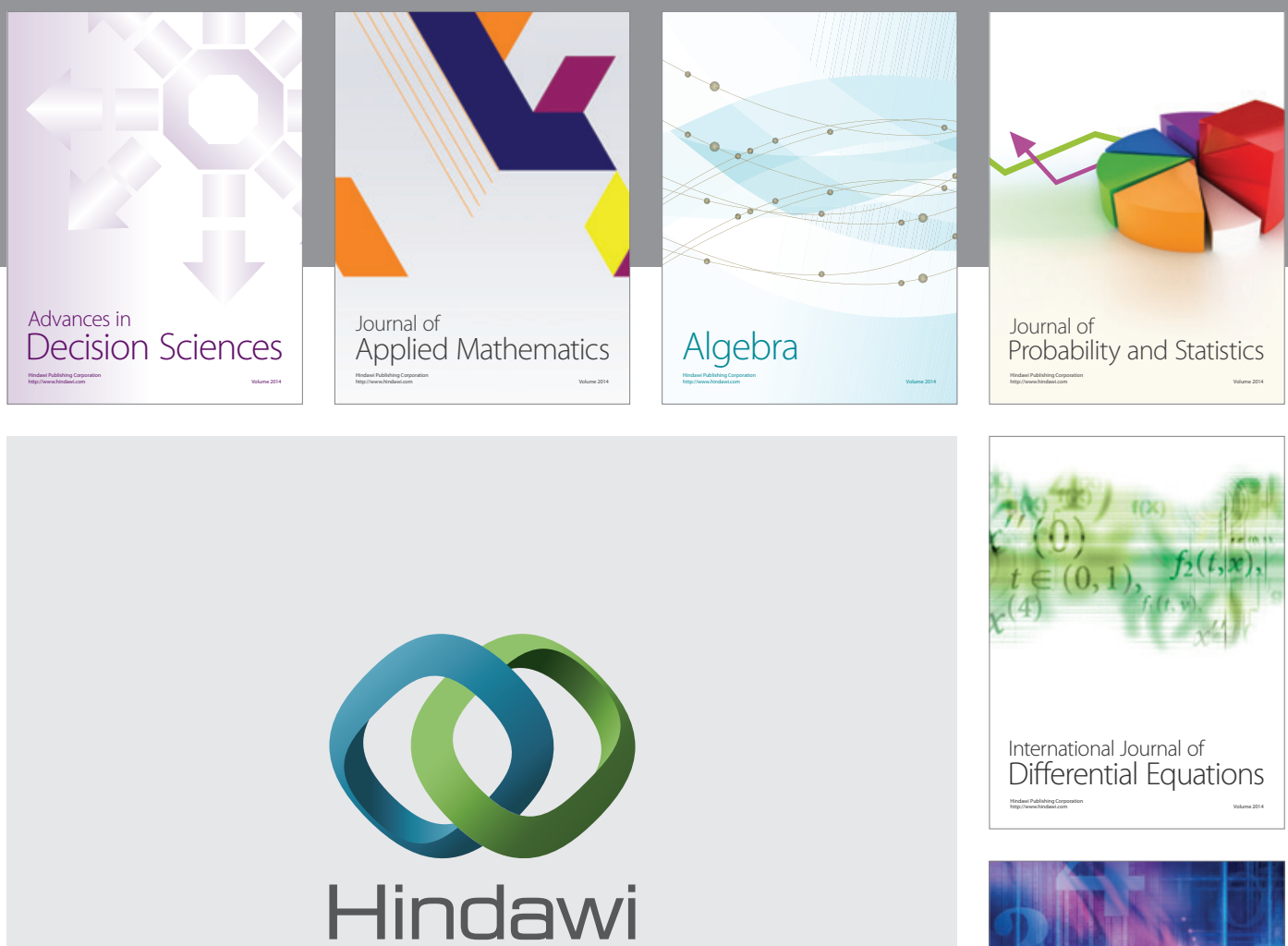

Submit your manuscripts at http://www.hindawi.com
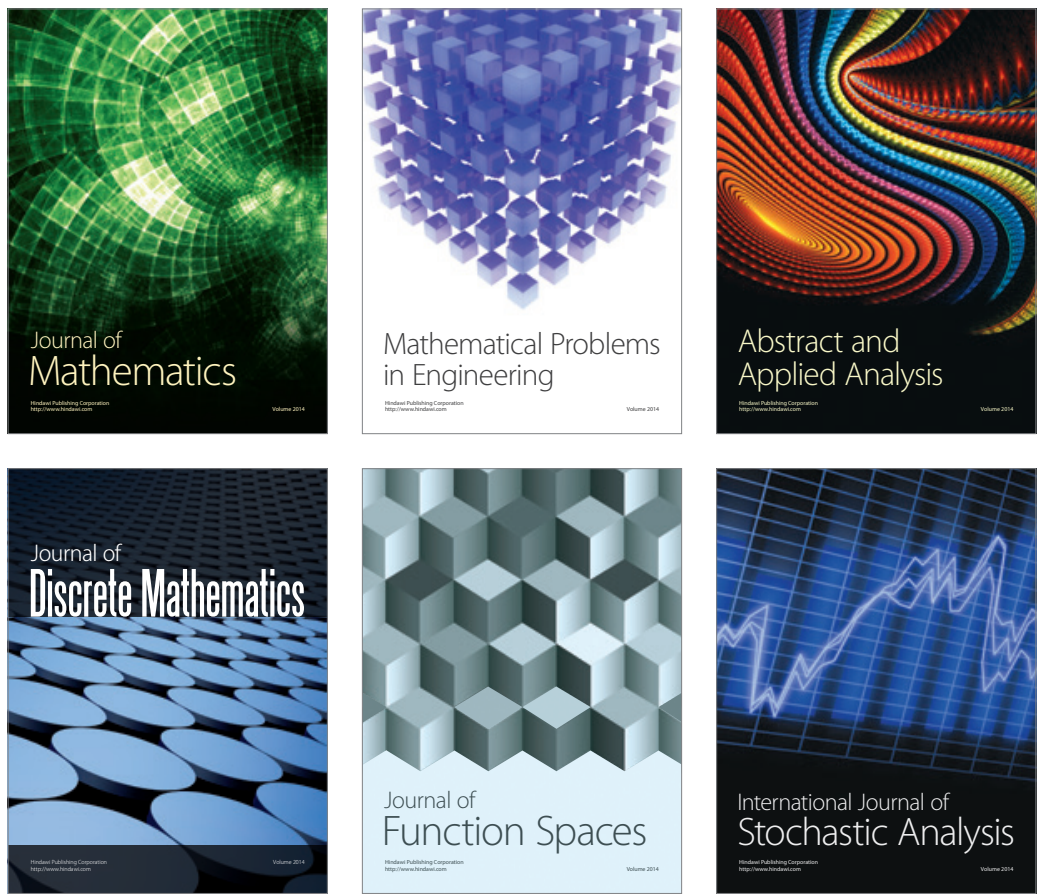

Journal of

Function Spaces

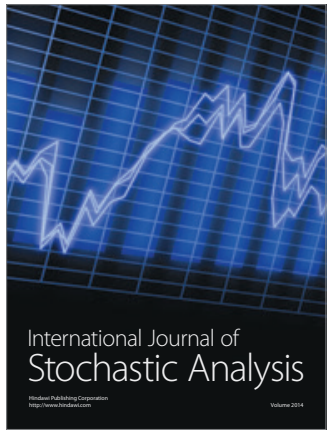

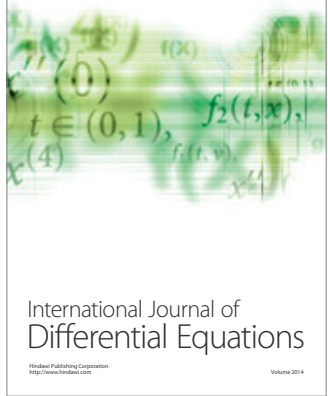
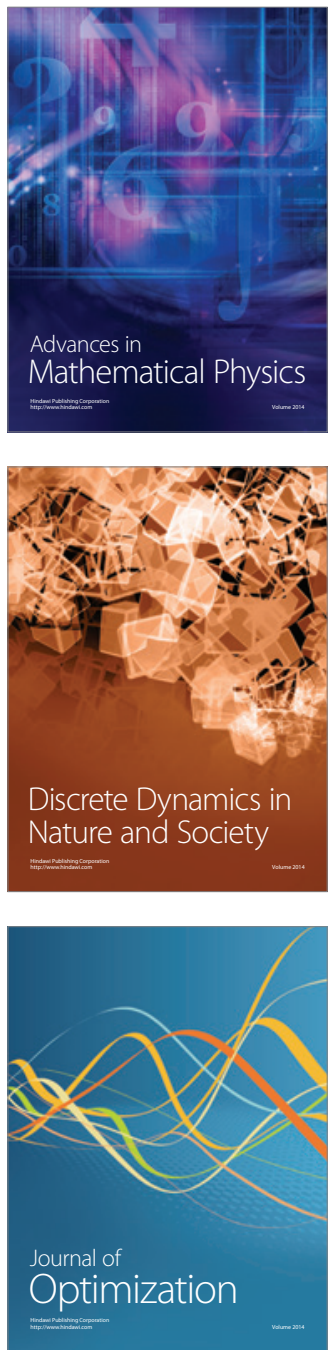